\title{
El régimen semántico de la afectividad en Sein und Zeit. Una interpretación semiótica de M. Heidegger
}

Adrián Bertorello*

Resumen: La diferencia semántica entre la disposición afectiva y la comprensión en Sein und Zeit radica en que el sentido inherente a la afectividad es del orden de lo continuo. En cambio, el sentido de la comprensión es del orden de lo discreto. El régimen de lo continuo se caracteriza por ser un campo de fuerzas que organiza el sentido de acuerdo a una continuidad gradiente. El régimen de lo discreto, por el contrario, organiza el sentido de acuerdo a una articulación de unidades significativas pragmáticas diferenciables que no se identifican con el lenguaje. De esta manera lo continuo y lo discreto puede ser reinterpretado como la fuerza y el significado. Así, entonces, el sentido de la disposición afectiva y el de la comprensión se distinguen por pertenecer a regímenes semánticos distintos. Los afectos pertenecen al dominio de la fuerza y la comprensión pertenece al campo del significado (Bedeutung).

Palabra clave: Afectividad, comprensión, fuerza, significado, Sein und Zeit, M. Heidegger.

Abstract: The semantic distinction between Disposedness and Understanding on Sein und Zeit can be described as the difference between the continuous (Disposedness) and the discrete (Understanding). The continuous domain organizes the space of meaning as strength field and a gradient polarization. In contrast the discrete domain organizes the space of meaning as an articulation of pragmatic meaningful and differential units. These units on Sein und Zeit don't identify with the language. So, the continuous and the discrete can be reinterpreted as strength and meaning. In this way, the space of meaning has two diverse domains: Disposedness belongs to the strength regime and Understanding to meaning (Bedeutung) field.

Key Words: Affectivity, understanding, strength, meaning, Sein und Zeit, M. Heidegger.

\footnotetext{
* Doctor en Filosofía y Magister en Análisis del Discurso por la Universidad de Buenos Aires. Investigador Independiente del Consejo Nacional de Investigaciones Científicas y Técnicas (CONICET). Lugar de Trabajo: Centro de Estudios Filosóficos "Eugenio Pucciarelli" de la Academia Nacional de Ciencias de Buenos Aires (ANCBA). Dirección electrónica: adrianbertorello@gmail.com
} 


\section{Planteamiento del problema: semiótica de los afectos y semiótica de la comprensión}

Una de las tesis más llamativas de Sein und Zeit es la relación entre el concepto de sentido y los estados de ánimo. Pareciera que los afectos son incapaces de producir efectos de sentido. O para decirlo de una manera exagerada: el sentido y los afectos, si bien son cooriginarios, pertenecen a regímenes distintos dentro de la estructura de la apertura constitutiva del Dasein. El sentido como fundamento de la interpretación es una noción que pertenece a la comprensión. Los afectos, por su parte, tienen su tratamiento temático en la disposición afectiva que, como existenciario (existenzial), se distingue de la comprensión. Si nos atenemos a la significación estricta del concepto de sentido, tendríamos que decir que los temples de ánimo están por fuera del sentido. Ciertamente que no son aquello que se opone al sentido. Heidegger alude de manera explícita al contrasentido cuando se refiere a los fenómenos naturales que irrumpen con violencia en el mundo. La disposición afectiva estaría, de acuerdo con esta primera lectura, por fuera del régimen del sentido.

Esta tesis resulta más llamativa cuando se la compara con otros abordajes teóricos de la afectividad donde el concepto de sentido es una noción que incluye tanto a la inteligibilidad de la dimensión pragmática de la comprensión como a la dimensión pasiva de los afectos. Así es abordado en la semiótica de las pasiones de A. Greimas y J. Fontanille. A diferencia de este modo de tratamiento, Heidegger circunscribe el sentido sólo al punto de vista activo de la comprensión. La pasividad característica de la disposición afectiva no se rige por la estructura del sentido. Sin embargo, en un breve pasaje del acápite 29 de Sein und Zeit el filósofo alemán afirma: "Y sólo porque los 'sentidos' [Sinne] pertenecen ontológicamente a un ente que tiene el modo de ser del ser en el mundo en disposición afectiva, pueden ser 'tocados' y 'tener sentido para' [Sinn haben für], de modo tal que lo que toca se muestra en la afección". 1

En este texto aparece un uso distinto del término sentido (Sinn). No designa las pre-condiciones de la interpretación, sino que se refiere a la afección. "Tener sentido para" significa "ser afectado por". La disposición

${ }^{1}$ Heidegger, M., Sein und Zeit, Tübingen, Max Niemeyer, 1986, p. 137. 
afectiva hace posible que algo se muestre en los sentidos como "lo que nos toca o afecta". Si bien Heidegger en Sein und Zeit restringe el concepto de sentido sólo a la comprensión, se puede decir, a partir de este pasaje, que la disposición afectiva se rige por una modalidad específica del sentido que se distingue del tener, ver y concebir previos. De esta cita se sigue necesariamente una pregunta: ¿en qué se distingue el sentido de la comprensión del sentido de la disposición afectiva?

En este trabajo querría mostrar la diferencia entre el sentido de la afección y el sentido de la comprensión. Como esta temática está sólo insinuada en el texto heideggeriano, intentaré explicitarla conceptualmente mediante un análisis que utiliza algunas herramientas de la semiótica de las pasiones de Greimas y Fontanille. ${ }^{2}$ La tesis que intentaré justificar se puede resumir de la siguiente manera: la diferencia semántica entre la disposición afectiva y la comprensión radica en que el sentido inherente a la afectividad es del orden de lo continuo. En cambio, el sentido de la comprensión es del orden de lo discreto. El régimen de lo continuo se caracteriza por ser un campo de fuerzas que organiza el sentido de acuerdo a una continuidad gradiente. El régimen de lo discreto, por el contrario, organiza el sentido de acuerdo a una articulación de unidades significativas pragmáticas diferenciables que no se identifican con el lenguaje. De esta manera lo continuo y lo discreto puede ser reinterpretado como la fuerza y el significado. Así, entonces, el sentido de la disposición afectiva y el de la comprensión se distinguen por pertenecer a regímenes semánticos distintos. Los afectos pertenecen al dominio de la fuerza. En cambio, la comprensión pertenece al campo del significado (Bedeutung).

El artículo tiene tres partes. En la primera retomo la última parte de un trabajo mío publicado en 2014 cuyo título es El principio de inmanencia y la diferencia ontológica. ${ }^{3} \mathrm{Al}$ final de ese texto había analizado los rasgos semánticos de una noción que Heidegger introdujo en 1919 y que luego nunca más retomó, a saber, el concepto de lo premundano (das Vorweltliche). Esta instancia es del orden de lo continuo y de la fuerza. En la segunda parte del presente trabajo muestro cómo los rasgos de lo premundano del año 1919 pasan a describir la

\footnotetext{
2 Greimas, A. y Fontanille, J., Semiótica de las pasiones, México, FCE, 2002.

3 Bertorello, A. "El principio de la inmanencia y la diferencia ontológica", Tópicos del Seminario 31, enero-junio 2014, pp. 175-193.
} 
disposición afectiva (Befindlichkeit) de Sein und Zeit (1927). La finalidad que persigo es destacar que, si bien el término "premundano" desaparece del vocabulario de $S$ ein und Zeit, sus rasgos descriptivos continúan en la concepción de la disposición afectiva. Ella es la que asume la condición de ser una instancia del orden de lo continuo y de la fuerza. Por último, en la tercera parte, intento justificar cómo se puede vincular un régimen semántico continuo con otro discreto. Es decir, cómo se vincula una semiótica de los afectos con una semiótica de la comprensión. Para ello tomo como punto de partida del análisis el concepto que, en Sein und Zeit, expresa la modalidad propia de la existencia: "el querer tener conciencia" (das Gewissen haben wollen).

\section{Lo premundano como instancia tensiva y continua del mundo}

En el trabajo Elprincipio de inmanencia y la diferencia ontológica abordé por primera vez la relación entre lo continuo y lo discreto en el pensamiento de Martin Heidegger. En aquel entonces mi reflexión se ceñía al concepto de lo premundano (das Vorweltliche) que aparece en la primera lección que Heidegger dictó en la Universidad de Freiburg cuando era asistente de Husserl: die Idee der Philosophie und der Weltanaschunng. Voy a tomar como punto de partida el análisis final de aquel trabajo para sentar las bases argumentativas de la interpretación que, en el presente artículo, va a centrarse en Sein und Zeit. En este punto me limito simplemente a realizar una síntesis de aquellas ideas que analicé detalladamente en mi trabajo anterior. Se trata de tres ideas principales.

La primera de ellas radica en que un régimen semántico discreto se caracteriza porque posee unidades diferenciables y articuladas entre sí. En cambio, un régimen continuo carece de unidades discretas. Lo continuo se mueve en el orden de las diferencias de grado. De acuerdo a esta distinción se puede afirmar que en la filosofía de Sein und Zeit el concepto de mundo (Welt), significiatividad (Bedeutsamkeit) y sentido (Sinn) corresponden a un régimen discreto. Cada uno de estos existenciarios describe la articulación de unidades discretas. En el caso del mundo esas unidades diferenciables y articuladas entre sí son: el "por mor del que" (Worumwillen), el "para" (Umæu), el "para qué" (Wozu), "en qué del dejar ser en respectividad" (Wobei des Bewendenlassens) y el "con qué de la condición respectiva" (Womit des Bewandtnis). Nuestra tesis es que la disposición afectiva (Befindlichkeit) corresponde a un régimen continuo. 
Pero esta afirmación la abordaré en el próximo punto. Lo que aquí quiero destacar es que cuando Heidegger en el año 1919 habla de una instancia anterior al mundo, se está refiriendo a un régimen semántico del orden de la fuerza y lo continuo. La disposición afectiva es la heredera conceptual de lo premundano.

La segunda idea consiste en que lo premundano se vincula con el mundo como lo potencial con lo actual. Lo premundano es una precondición del mundo. Se trata de la más alta potencialidad de la vida (die böchste Potentialität des Lebens) ${ }^{4} \mathrm{El}$ recorrido que va de lo premundano al mundo puede ser descrito como el pasaje de lo continuo a lo discreto. En efecto, lo premundano es aquello que carece de articulación significativa, pero que se direcciona y apunta al mundo: "[El algo fenomenológico] se extiende hacia la esfera de la vida en la que aún no está nada diferenciado, aún no hay nada mundano". $5 \mathrm{La}$ direccionalidad de lo premundano hacia el mundo expresa justamente que no se trata de algo así como una instancia alosemiótica, es decir, sin sentido, sino que, por el contrario, da cuenta de una orientación semántica que no es otra cosa que la intencionalidad. Se trata de una intencionalidad más originaria que la de la totalidad de significados del mundo.

La tercera idea presenta a lo premundano como una precondición de la articulación significativa del mundo que se mueve en el plano de la fuerza y la tensión. Lo premundano expresa una intensidad vital que se organiza de acuerdo a una polarización gradiente. Ello puede apreciarse cuando Heidegger afirma que podemos experimentar lo premundano no sólo cuando pasamos de un mundo a otro, sino fundamentalmente en los momentos de mayor intensidad vital (in Momenten besonders intensiven Lebens). ${ }^{6}$ Aquí se puede apreciar claramente que lo premundano es una fuerza, una tensión, que se rige de acuerdo a una continuidad gradiente.

De esta caracterización de lo premundano como una precondición de la significación (mundo), que tiene el rasgo de la fuerza y lo continuo,

${ }^{4}$ Heidegger, M., Die Idee der Philosopbie und der Weltanschaunng en Zur Bestimmung der Philosophie, Frankfurt am Main, Vittorio Klostermann, 1999, p. 115.

${ }^{5}$ Ibid., p. 218.

${ }^{6}$ Ibid., p. 115. 
concluíamos que se trataba de una instancia pasional, afectiva. Lo premundano y el mundo se vinculan como la fuerza y el significado.

\section{Lo premundano y la disposición afectiva (Befindlichkeit)}

La ausencia de articulación significativa, la continuidad gradiente de la intensidad y la fuerza, como así también la precedencia de lo continuo y tensivo frente a lo discreto son algunos de los rasgos con los que la semiótica greimasiana caracteriza la dimensión afectiva del sentido. Si bien en el texto de 1919 no hay una referencia explícita al carácter afectivo de lo premundano, ${ }^{7}$ se puede inferir esta pertenencia a partir de los rasgos descriptivos del mismo. Ciertamente que esta inferencia no se puede llevar a cabo sólo a partir de los elementos textuales. Es necesario utilizar como criterio de lectura algunas nociones de la semiótica para poder sacar a la luz el componente pasional de lo premundano.

Me parece que la afirmación heideggeriana del año 1919 de una instancia semántica anterior a la articulación significativa del mundo, es decir, la idea de que hay un protosentido afectivo y un sentido diferenciado inherente al mundo queda recogida en Sein und Zeit en dos caracteres de la disposición afectiva. El rasgo de lo premundano, que más arriba designé como la apertura de una intencionalidad originaria, es decir, con el hecho de que lo premundano es una tendencia y orientación hacia el mundo, aparece recogido en Sein und Zeit en el segundo aspecto de la disposición afectiva: "El estado de ánimo ha abierto en cada caso ya el ser en el mundo como un todo y hace posible en primer lugar un orientarse a..." 8

La afectividad se presenta en la cita como el a priori ontológico de la intencionalidad. La indicación de prioridad que establece el "hace posible en primer lugar" (allererst) remite a la caracterización de lo premundano como una precondición de la vivencia del mundo circundante. La disposición afectiva abre un horizonte tensivo, un espacio orientado de sentido cualificado por

\footnotetext{
7 Para un enfoque del lugar metodológico que tiene el concepto de lo premundano, véase Vigo, A., "Fenomenología y hermenéutica en las primeras lecciones de Friburgo (1919-1921)" en Arqueología y aleteiología, Biblos, Buenos Aires, 2008, pp. 231-257.

${ }^{8}$ Heidegger, M., Sein und Zeit, ed. cit., p. 137.
} 
fuerzas e intensidades, que cooriginariamente se reticula en un plexo de significaciones. El horizonte tensivo de la afectividad se organiza semánticamente en torno a una polarización que Heidegger describe como conversión (Ankehr) y aversión (Abkehr). Estos términos describen los polos opuestos de la facticidad de la condición de arrojado. La condición de arrojado (Geworfenheit) es un existenciario del orden de la fuerza que puede ser concebido como un metaquerer ${ }^{9}$ (en la medida en que es anterior a toda determinación volitiva). Que se trata justamente de una dimensión en la que se ponen en juego fuerzas, aparece claramente cuando Heidegger afirma que el modo inmediato de la facticidad es el de la represión (abgedrängt). Si bien la aversión y su polo contrario admiten una continuidad gradiente, no pareciera que el texto heideggeriano explora esta posibilidad.

De todos los estados de ánimo es la angustia la que muestra de un modo más nítido esta relación entre un sentido concebido bajo el régimen de lo continuo y la fuerza y otro sentido que se rige por la articulación significativa de unidades discretas. La nada y el "ningún lugar" característicos de este temple anímico muestran al análisis fenomenológico que el mundo familiar se hunde en la insignificatividad (Unbedeutsamkeit). ${ }^{10} \mathrm{La}$ metáfora del hundimiento del mundo (herabsinken) ilustra claramente que lo que revela la angustia es la pérdida de la articulación significativa reticular a partir de la cual el Dasein comprende los entes. Por eso dice Heidegger que la insignificatividad del mundo "puede poner en libertad un ente en el carácter de la falta de condición respectiva (Unbewandtnis)". ${ }^{11}$ Las condiciones de inteligibilidad del ente se desarticulan. La nada es la expresión de la continuidad semántica, es decir, de la ausencia de diferencias. Al no poder estar en condición respectiva respecto del ente, al no poder articularlo comprensivamente en una red de relaciones significativas, también el ente se hunde en una continuidad indiferenciada y se muestra como una vacía impasibilidad (leere Erbarmungslosigkeit). ${ }^{12}$

La impasibilidad del ente está relacionada con el tercer rasgo de la disposición afectiva que omití más arriba. Los estados de ánimo abren un

\footnotetext{
${ }^{9}$ Ibid., p. 135

${ }^{10}$ Ibid., p. 343

11 Ibid.

12 Ibid.
} 
horizonte tensivo continuo polarizado de acuerdo a las dos fuerzas de la aversión y la conversión. Este espacio dinámico no perceptivo, sino afectivo, es la condición de posibilidad de la pasividad del Dasein. Los entes pueden afectarlo porque la disposición afectiva abre ese horizonte. La angustia como estado de ánimo destacado modifica el espacio tensivo de modo tal que el Dasein se torna impasible ante el ente.

Así, entonces, se puede concluir que el concepto de lo premundado como una instancia semántica anterior a la articulación significativa del mundo queda recogida en Sein und Zeit en la disposición afectiva. Los temples de ánimo no son del orden de lo discreto, sino que su sentido específico se desarrolla en el plano de una continuidad gradiente. De esta manera se puede apreciar con claridad que los afectos están por fuera del sentido concebido como articulación. Sin embargo, no puede afirmarse que estén por fuera del espacio del sentido. Por el contrario, como acabamos de mostrar, tienen una estructura semántica específica. El interrogante que surge de esta caracterización es cómo se vinculan dos regímenes heterogéneos como lo continuo y lo discreto. Una posibilidad exploratoria podría ser analizar este problema desde un punto de vista modal.

\section{El vínculo entre lo continuo y lo discreto desde una perspectiva modal: querer y metaquerer}

En un trabajo anterior ${ }^{13}$ traté el sentido del abordaje heideggeriano de las modalidades. Sostuve la tesis de que el marco apropiado para una correcta comprensión de los términos modales en Sein und Zeit no era la lógica modal, sino una consideración semiótico-narrativa. Apoyaba mi argumentación en la lectura que $O$. Becker hacía de la ontología heideggeriana en su libro Untersuchungen über den Modalkalkül. Según este autor, la ontología de Sein und Zeit se resiste a un tratamiento lógico modal a raíz de que el segundo axioma de la lógica modal, a saber, la necesidad determina la posibilidad, carece de validez dentro de la ontología heideggeriana, para la cual, la posibilidad es más

13 Bertorello, A., "Una interpretación semiótico-narrativa del sistema de las modalidades en Sein und Zeit' en Studia Heideggeriana 2, Editorial Teseo, Buenos Aires, 2012, pp. 57-70. 
originaria que la necesidad. Esta inversión de la jerarquía de las modalidades operado en el pensamiento de Heidegger hace imposible un análisis estrictamente lógico. ${ }^{14}$

Sin embargo, sostenía en aquel trabajo que ello no tenía como consecuencia la impo-sibilidad de un tratamiento sistemático de las modalidades. ${ }^{15}$ Para poder sacar a la luz el sistema modal de Sein und Zeit era más adecuado hacer una lectura semiótico-narrativa. La razón de ello radica en que el análisis heideggeriano del lógos no se sitúa en un nivel lógico-axiomático independiente de las condiciones fácticas de la enunciación, sino más bien se coloca en un punto de vista genético que intenta mostrar cómo los conceptos lógicos se derivan de la existencia fáctica del Dasein.

Que el lugar teórico para pensar las relaciones entre lo continuo y lo discreto sea las modalidades se puede advertir claramente en las relaciones que existen entre lo que, en el punto tres, denominé como un "metaquerer" y el querer específico de la propiedad de la existencia. Para finalizar este trabajo me referiré a este problema sólo de manera exploratoria. El objetivo no es hacer una exposición exhaustiva de los vínculos entre lo continuo y lo discreto, sino tan sólo mostrar el lugar al que hay que dirigir la mirada para llevar a cabo un análisis de esta cuestión.

La expresión "metaquerer" procede de Semiótica de las pasiones. Greimas y Fontanille la utilizan para dar cuenta de la relación entre las precondiciones de la significación y los diversos posicionamientos del sujeto. El actante sujeto, para llevar a cabo una transformación narrativa debe, posicionarse frente a un objeto que reviste un cierto valor para él. De esta manera el sujeto es portador del predicado modal del querer. ${ }^{16}$ Ahora bien, este predicado constitutivo de un aspecto de la identidad del sujeto puede comparecer como tal porque de alguna manera se funda en una condición anterior a la distinción sujeto-objeto.

14 Véase Becker, O., Untersuchungen über den Modalkalkül, Meisenheim am Glan, Westkulturverlag Anton Hain, 1952, p. 70 y ss.

${ }_{15}$ Para una interpretación sistemática de las modalidades en Heidegger desde un punto de vista lógico, véase Ramos dos Reis, R., Aspectos da modalidade. A noção de possibilidade na Fenomenologia Hermenêtica. Viaverita, Rio de Janeiro, 2014

${ }^{16}$ Fontanille, J., Semiótica del discurso, Lima, Fondo Editorial de la Universidad de Lima, 2006, pp. 142 y ss. 
Esta condición de posibilidad, que hace que un sujeto pueda querer un objeto, es lo que Greimas y Fontanille llaman las "precondiciones de la significación". Se trata de una instancia, que es anterior a la lógica de la acción humana (propia de las transformaciones narrativas) y que tiene un carácter pasional. Un determinado actante sujeto puede querer un objeto de valor porque este vínculo intencional está fundado en una instancia afectiva previa que abre la posibilidad para que pueda vincularse volitivamente con algo. Las pasiones dan cuenta, para la semiótica narrativa, de una intencionalidad más originaria sobre la que se funda la intencionalidad de la acción. Nuestro análisis sobre el carácter premundano tomaba como punto de partida esta idea. Lo que quiero destacar ahora es que las precondiciones de la significación tienen un valor modal. Se trata de un "metaquerer" cuyo sentido cobra ahora una significación más precisa: el querer del sujeto, que establece su vínculo intencional con un objeto, tiene su condición de posibilidad en un querer anterior a la distinción semiótica entre sujeto y objeto. Por ello se trata de un metaquerer de índole pasional.

Este vínculo entre un querer que se le imputa a un sujeto práctico que obra, y otro que se le atribuye a una instancia presubjetiva y afectiva, puede servir como modelo para comprender la expresión con la que Heidegger, en Sein und Zeit, describe el modo propio de la existencia, a saber, "el querer tener conciencia" (Gewissen haben wollen). Esta expresión estructura los tres existenciarios que articulan cooriginariamente la apertura (Erschlossenheit): la comprensión (Verstehen), la disposición afectiva (Befindlichkeit) y el discurso (Rede). El querer da cuenta de la comprensión, ya que es la modificación propia del "por mor de qué" (Worumwillen). La conciencia, por su parte, recubre el campo del discurso y la disposición afectiva. La articulación discursiva de la conciencia es el callar (Verschwiegenheit) y su temple de ánimo específico es la angustia.

La expresión completa "querer tener conciencia" estructura cooriginariamente lo continuo y lo discreto. En efecto, el callar se presenta como la ausencia de articulación fonética, como un continuum privativo. La inhospitalidad (Unheimlichkeit) de la angustia, como vimos más arriba, expresa también otro continuum privativo, a saber, la ausencia de articulación significativa. El querer es, por su parte, un verbo modal que rige, modaliza, el continumm del silencio y la inhospitalidad. Como modificación propia del 
Worumwillen comprensor pertenece al dominio de lo discreto. En efecto, da cuenta de uno de los momentos que articula la significatividad (Bedentsamkeit).

Ahora bien, el verbo modal, que designa el momento comprensivo de la propiedad, guarda una relación circular con una instancia afectiva que, de alguna manera, puede ser equiparada con el metaquerer de la semiótica. Esta afirmación se funda en los análisis anteriores donde mostramos que el concepto de lo premundano queda recogido estructuralmente en la disposición afectiva. En cuanto la disposición afectiva abre por primera vez la dirección intencional al mundo y la condición de arrojado, se puede afirmar que el querer comprensor quiere lo abierto en los temples de ánimo. Pero a su vez, lo abierto en la disposición afectiva se presenta como una fuerza del orden de lo continuo que puede ser interpretada como un metaquerer en el sentido que Greimas y Fontanille le da a este término. Esta última afirmación resulta particularmente evidente en el caso de la propiedad de la existencia. Tomo como punto de partida el siguiente pasaje de Sein und Zeit: "Comprender la llamada es el elegir [Wählen] no la conciencia, que como tal no puede ser elegida. Es elegido el 'querer tener conciencia' [das Gewissen haben wollen] como el estar libre para el más propio ser culpable. Comprender la interpelación significa: querer-tenerconciencia [Gewissen-baben-wollen]". ${ }^{17}$

De esta cita se sigue que el verbo modal de la comprensión propia tiene que ser in-terpretado como una elección. De esta manera, el querer cobra una fisonomía muy precisa. Se trata de un acto libre. Ahora bien, el contenido de la elección es el ser-culpable del Dasein. La culpabilidad entendida como el fundamento de una negatividad (Grundsein einer Nichtigkeit) ${ }^{18}$ atraviesa todo el ser del Dasein: la existencia, la facticidad y la caída ${ }^{19}$.

La relación peculiar entre un querer como elección libre y un metaquerer como la precondición semántico-afectiva-continua-pulsional se puede ver muy claramente en la facticidad. Me limitaré a citar un breve pasaje donde Heidegger describe la condición de arrojado como un presupuesto afectivo de la elección. La idea central es que la facticidad tiene el carácter del fundamento (Grund) no elegido desde el cual el Dasein existe. La condición de arrojado concebida como

${ }^{17}$ Heidegger, M., Sein und Zeit, p. 288.

${ }^{18}$ Ibid., p. 283

${ }^{19}$ Ibid., p. 284 
un fundamento negativo puede ser interpretada como la instancia presignificativa que la semiótica narrativa denomina como metaquerer:

Siendo, el Dasein es un ente arrojado, no se ha puesto a sí mismo en su Ahí. Siendo, está determinado como un poder ser, que se pertenece a sí mismo y que, sin embargo, en tanto él mismo no se ha dado a su propiedad (...) Estando entregado a ser este determinado ente -única forma como él puede existir como el ente que él es- el Dasein es -existiendo- el fundamento de su poder ser. Si bien él por sí mismo no ha puesto el fundamento, descansa en una pesadez que el estado de ánimo [Stimmung] le revela como una carga. $^{20}$

En la primera cita, Heidegger afirmaba que la conciencia como tal no puede ser elegida. El contenido de la elección es el "tener conciencia". La razón por la que la conciencia no puede ser elegida radica precisamente en que abre una instancia, a saber, la culpabilidad, que es el fundamento no electivo de toda elección. En virtud de que uno de los rasgos de ese fundamento negativo es la condición de arrojado se puede afirmar que cumple la función de un metaquerer presupuesto en todo querer.

Recibido: 11/2016; aceptado: 12/2016

${ }^{20}$ Ibid. 